\title{
Zweiter Beitrag
}

\section{zur \\ Kenntnis der Diplopodenfauna}

\author{
der Schweiz
}

von

\section{Dr H. ROTHENBÜ HLER,}

BERN.

Hierzu Tafel 13.

Die nachstehenden Mitteilungen bilden die Fortsetzung zu meiner ersten, in der Revue suisse de Zoologie erschienenen Arbeit über die Myriapodenfauna der Schweiz und enthalten die Resultate meiner Untersuchungen im Jahre 1899. Dem vorhandenen Material entsprechend wurden nur die Diplopoden berücksichtigt. Im Vergleich zur Grösse der noch zu lösenden Aufgabe - genaue Kenntnis der schweizerischen Myriapodenfauna können die gewonnenen Ergebnisse nur als kleiner Schritt nach dem Ziele hin bezeichnet werden.

In Bezug auf die Entdeckung neuer Arten kann man allerdings schon jetzt mit Sicherheit annehmen, dass eine grosse Anzahl solcher im Gebiet der Schweiz nicht mehr vorhanden ist. Immerhin werden der Süden und Osten unseres Landes, die Täler des Wallis und Tessin und diejenigen Graubündens noch einiges Neue liefern, während dies von Gegenden mit vorwiegendem Kulturland nicht zu erwarten ist. Auch der Jura wird kaum noch Neues enthalten. 
In anderer Beziehung aber bietet sich dem Forscher noch reichlich Gelegenheit zur Bethätigung. Für eine ganze Anzahl bekannter Arten ist das Vorkommen in der Schweiz zwar wahrscheinlich, aber nicht sicher erwiesen. Es betrifft dies Formen, welche für angrenzende Gebiete, z. B. Deutschland und Frankreich, bekannt sind, und von denen nicht anzunehmen ist, dass sie das dazwischen liegende Terrain der Schweiz meiden sollten. Von keiner endemischen Art, beispielsweise Polydesmus helveticus, sind die Grenzen ihres Verbreitungsgebietes genauer erforscht; ebenso wenig kennt man die Verbreitungsgrenzen von Arten, welche vicariirende genannt werden, z. B. Polydesmus complanatus und illyricus.

Ausser diesen tiergeographischen Beobachtungen bietet die Biologie der Myriapoden eine solche Fülle von interessanten Aufgaben, dass Jahre zu deren Lösung erforderlich sind.

Wünschenswert und höchst erfreulich wäre es, wenn sich unter den schweizerischen Entomologen oder andern Naturfreunden Männer fänden, welche die Arbeit des Sammelns übernehmen würden, indem dadurch die Kenntnis der schweizerischen Myriapodenfauna ungemein viel rascher als durch die Bemühungen eines Einzelnen gefördert würde.

Den Herren $\mathrm{D}^{\mathrm{r}}$ J. CARL und $\mathrm{D}^{\mathrm{r}}$ Th. STECK, welche mich durch Ueberlassung wertvollen Materials unterstützten, sei an dieser Stelle für ihre Mithülfe mein wärmster Dank ausgesprochen.

\section{Familie GLOMERID $\nRightarrow$ Leach ${ }^{1}$.}

$$
\text { Gattung Glomeris. }
$$

Es kann mit Sicherheit angenommen werden, dass ausser den bereits bekannten Arten sich in der Schweiz keine neuen

\footnotetext{
${ }^{1}$ Vergleiche hiezu: Verheff, Ueber Glomeriden und Atтens, Ueber die Färbung von Glomeris; beides im "Archiv für Naturgeschichte ", 1900, Bd. I, Heft 3.
} 
Glomeriden finden werden ${ }^{1}$. Meine ergänzenden Bemerkungen über diese Familie beschränken sich auf die Wohnorts-Verhältnisse derselben.

\section{Glomeris conspersa C. Koch.}

In hügeligem Gelände weithin durch das mittlere Europa verbreitet und im Alpengebiet bis in die entlegensten Hochtäler vorgedrungen; sie fehlt auch dem Südabhang der Alpen und der Lombardei nicht. Aus der Umgebung von Lugano und vom Monte San Salvatore besitze ich mehrere Tiere von 16-18 mm. Länge, also von recht bedeutender Grösse.

Auffallend ist die Erscheinung, dass Gl. conspersa in denjenigen Alpenthälern, wo Gl. transalpina häufig ist, recht selten vorkommt, auch wenn günstige Existenzbedingungen für sie vorhanden sind. Die Höhenlage kann hiefür nicht ein genügender Grund sein, da sie bis zu 2000 m. Höhe recht gut fortkommt; wahrscheinlich aber bieten ihr die mit Laubhölzern nur wenig gemischten Tannenwälder im ganzen nicht zusagende Nahrung. Daraus erklärt sich, dass das Tier in diesen Tälern sich auch

${ }^{1}$ Anmerkung: Man vergleiche hiezu : H. FAEs, Contribution à l'étude des Myriapodes. Bulletin de la société vaudoise des sciences naturelles, No 136, Juni 1900. Dort sind als für die Schweiz neue Arten aufgezählt:

1. Glomeris cingulata Koch.

2. Glomeris pustulata Latr.

3. Glomeris guttata Risso.

4. Glomeris tridentina Latzel.

Hiezu bemerke ich folgendes:

ad 1. Gl. cingulata $=G l$. transalpina var. cingulata, was sich aus der Berücksichtigung der neuern Litteratur ergibt.

ad 2. Ist möglich, könnte aber auch eine Farbenvarietät von Gl. ornata sein.

ad 3. Diese mysteriöse Art ist seit ihrer Entdeckung nie mehr mit Sicherheit erkannt worden. Der Verfasser hätte daher mit einer guten Diagnose der Wissenschaft einen grossen Dienst geleistet. Solange dies nicht geschieht, ist die Aufstellung dieser Art unter den schweizerischen Myriapoden als irrtümlich zurückzuweisen.

ad. 4 . Gl. tridentina $=G l$. connexa var. valesiaca $(?=$ var. ligurica Latzel).

Von den vier als neu im Gebiet nachgewiesenen Arten hat nur Gl. pustulata einige Wahrscheinlichkeit als neue Art für sich. 
an günstigen Stellen, z. B. in Laubholzgebüschen, selten vorfindet.

\section{Glomeris hexasticha Brandt.}

Diese Art zeigt für östliche und westliche Gegenden einen bemerkenswerten allgemeinen Unterschied in der Zeichnung. Nach LATzEL ist «nicht selten » neben den übrigen sechs ein siebenter Längsstreif von Flecken auf der Rückenmitte ausgebildet. Dieser Ausnahmefall für östliche wird für die mehr westlichen Tiere zur Regel. Aus der mittleren und westlichen Schweiz ist mir nie eine Gl. hexasticha mit nur sechs wohl ausgebildeten Fleckenreihen vor Augen gekommen; immer ist der mediane Rückenstreif vorhanden, wenn überhaupt die Zeichnung deutlich, und die sämtlichen Streifen nicht verwischt sind. Auch gehört die Mehrzahl dieser Individuen zur Unterart intermedia oder trisulcata; die typische Form mit nur einer durchgehenden Brustschildfurche ist dagegen nur spärlich vertreten.

Schon in der Ostschweiz ändert sich dieses Verhältnis. Aus dem Engadin erhielt ich mehrere Stücke durch Herrn Dr CARL, welche sämtlich ohne Ausnahme der typischen Form angehören. Unter diesen sah ich auch zum ersten Mal Tiere mit sechs Längsstreifen und ohne die mediane Rückenlinie.

Es ergiebt sich daher, dass Glomeris hexasticha mit dem weitern Vordringen nach Westen die Tendenz zur regelmässigen Ausbildung des medianen Rückenstreifens zeigt.

\section{Glomeris transalpina C. Koch.}

Ihr hauptsächlichstes Verbreitungsgebiet sind die Centralalpen, und hier sowohl die Talsohle als auch die Berghänge bis zu $2500 \mathrm{~m}$ Höhe. Sie findet ihre Futterplätze vorzugsweise im Moos und in vermodernden Nadeln des Tannenwaldes, kommt aber auch auf Bergwiesen und Alpweiden gut fort, sobald die nötigen Gesteinstrümmer als Unterschlupfsorte vorhanden sind. 
An den bereits bekannten Orten, nämlich in den Tälern von Wallis, Tessin und Graubünden, kommt das Tier massenhaft vor, dagegen tritt es in den der Alpenkette im Norden vorgelagerten Voralpen sehr sporadisch auf, und es sind mir von da nur zwei Fundorte bekannt geworden, wovon der eine (Faulhornkette) bereits im meinem ersten Beitrag genannt wurde. Neuerdings fand ich zwei Exemplare des Tieres im Justistal oberhalb Merligen am Thunersee, an beiden Orten zusammen drei Stück. Der letztgenannte Ort dürfte jedenfalls nahe der nördlichen Verbreitungsgrenze der Art liegen.

Familie POLYDESMID E Leach.

Gattung Strongylosoma Brandt.

Strongylosoma italicum Latzel.

Zahlreiche Exemplare von Nyon am Genfersee; (Schlossgarten von Prangins unter Ziegelsteinen) alle von blassgrünlicher Färbung.

Humberts Angabe in den Myriapodes des environs de Genève über Strongylosoma pallipes ist irrtümlich und bezieht sich auf Strongylosoma italicum.

Gattung Polydesmus Brandt.

Polydesmus coriaceus Porat.

Fig. 6.

Sein Verbreitungsgebiet ist ein ungewöhnlich grosses, da er aus Schweden, Rheinpreussen (P. rhenanus Verh.) und von den Azoren bekannt ist. Dazu kommt als neuer Fundort Sitten im Wallis, wo ich die Tiere auf einer feuchten Wiese unter Steinen fand. Fig. 6 stellt einen Copulationsfuss eines dieser Tiere dar. Aus der Abbildung ist ersichtlich, dass der Hauptast (Innenast) nicht einfach « vogelkopfähnlich » endet, wie VERHGFF es dar- 
stellt; eher könnte man das Ende mit einem asymmetrisch gestalteten Hutpilze vergleichen, weil es auch nach der Seite des Nebenastes hin in einer kurzen Spitze vorspringt. Dieser Unterschied in der Zeichnung beweist jedoch noch nicht ohne weiteres einen Unterschied des betreffenden Organes, sondern kann auch durch die Lage des Präparates bedingt sein.

\section{Polydesmus subinteger Latzel.}

Ist nach meinen bisherigen Erfahrungen auf den südwestlichen Teil der Schweiz beschränkt. Von Nyon am Genfersee erhielt ich neuerdings eine Anzahl Tiere durch Herrn Dr CARL. Aus dem Wallis sind sie schon von früher her bekannt.

Anschliessend sei hier eine neue Unterart von $P$. subinteger aus den cottischen Alpen bekannt gemacht; dieses Gebiet gehört zwar politisch nicht der Schweiz, aber topographisch dem Alpengebiet an.

Polydesmus subinteger Latz. subsp. Steckii n. subsp ${ }^{1}$. Fig. 15.

Länge $18 \mathrm{~mm}$.

Die Körperbeschaffenheit stimmt mit subinteger überein. Die unterscheidenden Merkmale liegen in den Gonopoden oder Copulationsfüssen, Fig. 15. Der Hauptast ist von anderer Gestalt und viel dicker und länger als bei der Stammform. Er zeigt viel Aehnlichkeit mit einem menschlichen Fuss. Das in der Querrichtung verbreiterte Ende lässt drei durch flache, in der Längsrichtung verlaufende Rinnen entstandene Wülste erkennen, von denen der mittlere ein kurzes Hörnchen trägt. Am Ende sind Haarpolster und Samenblase deutlich sichtbar. Diesem Hauptast ist der Nẹbenast ziemlich eng angeschmiegt; er ist stärker gebogen als bei subinteger und im Verhältnis zum Hauptast

\footnotetext{
${ }^{1}$ Benannt nach dem Entdecker des Tieres, Herrn Dr Steck, Conservator am entomologischen Museum in Bern.
} 
kürzer als bei jenem. Der dreieckige Zahn auf der hohlen Innenseite sitzt nicht in der Mitte des Astes, sondern im Anfang des letzten Vierteils gegen das Ende hin.

Die Abweichungen im Bau der Gonopoden erweisen sich als constant und übereinstimmend bei Tieren von drei verschiedenen Orten; sie sind daher mehr als bloss variable Erscheinungen und verleihen dem Tiere den Charakter einer guten Unterart.

Fundort: Cottische Alpen und Tarantaise : Col Clapier 1600 m. Mont Albergian $2000^{\mathrm{m}}$. Col de la Vanoise $2100^{\mathrm{m}}$. Zusammen $5 \sigma^{7}$ und 3 ?.

\section{Polydesmus illyricus Verhœff.}

Länge $18-20^{\mathrm{mm}}$, Breite schwach $3 \mathrm{~mm}$.

Die Gonopoden entsprechen durchaus der Abbildung 207 in: AtTens, System der Polydesmiden. Die Kiele der fünf ersten Segmente sind mässig aufgebogen, die Pusteln auf der Oberseite der Kiele heben sich durch ihre hellere Färbung auffällig vom dunklern Grunde ab.

Ein Weibchen hatte am 9. Juli einen grossen Eierklumpen abgelegt.

Fundort : Engadin, Val Triazza, rechte Talseite, 2000 m Höhe, unter Rinde alter Baumstöcke. Alp Fetan 1500 m.

Es kann nicht überraschen, dass das Engadin nur von Polydesmus illyricus besiedelt ist, während complanatus anscheinend dort fehlt. Denn das Engadin als der oberste Teil des Inntales ist jedenfalls zum grossen Teile vom Tirol her mit Myriapoden bevölkert worden, und dort ist nur illyricus, nicht aber complanatus nachgewiesen. Dazu kommt der Umstand, dass der erstere mehr ein Bewohner der Berggelände ist, der letztere aber Ebenen und Hügelgelände vorzieht. Wenn wir trotzdem im Berner-Oberland und Wallis keine illyricus, wohl aber complanatus als ständige Polydesmiden antreffen, so hat dies seinen Grund darin, dass diese Täler von Norden und Westen her besiedelt wurden. 
Dem Vordringen des illyricus in diese Gegenden setzten die hohen Alpenpässe und -ketten aber unübersteigbare Schranken entgegen; die Frage, wo im Alpengebiet für illyricus die Westgrenze liege, muss erst noch untersucht werden. Es ist nicht unmöglich, dass er vom Engadin aus in einige westlicher gelegene Täler Graubündens hinübergestiegen ist.

\section{Polydesmus subulifer Brölemann.}

Wahrscheinlich erstreckt sich das Verbreitungsgebiet dieser Art auf den grössten Teil des südlichen Abfalls der Alpen; sicher nachgewiesen ist sie erst von zwei Orten; in den Tälern der Briance (Südwestfrankreich) entdeckte sie BröLemann, und am Monte San Salvatore bei Lugano fand ich ein Männchen dieser Art.

Polydesmus trunculus, den ich in meinem ersten Beitrag als neue Art aus dem Wallis beschrieb, muss bis zur Auffindung anderer gleich gebauter Tiere als besondere Art zweifelhaft bleiben und zwar wegen der ganz ungewöhnlichen Gestaltung der Copulationsfüsse. Graf ATTEMs, der die Freundlichkeit hatte, mein Originalexemplar und die Präparate einer Nachprüfung zu unterziehen, glaubt, dass es sich um einen Polydesmus complanatus handle, der durch irgend einen Zufall die Enden der Copulationsfüsse verlor. Diese Frage kann erst durch Auffindung weiterer Exemplare von "Polydesmus trunculus » entschieden werden.

\section{Familie CHORDEUMIDA C. Koch.}

Die Formenmannigfaltigkeit in der Chordeumidenfamilie ist ausserordentlich gross, und es ist keine geringe Arbeit, über dieses Formengewirre eine klare Uebersicht zu gewinnen. Eine solche besitzen wir gegenwärtig noch nicht, und daran ist in erster Linie der Umstand schuld, dass in Bezug auf das Hauptgruppirungsprincip unter den Forschern nicht Uebereinstimmung 
vorhanden war. Demzufolge können viele neubeschriebene Tiere nicht ins System eingereiht werden, weil die wichtigsten Merkmale gar keine oder nur ungenügende Berücksichtigung fanden. Als Hauptgruppirungsprincip müssen naturgemäss diejenigen Organe und Organgruppen bezeichnet werden, welche in ihrer Differenzirung und verschiedenartigen Ausgestaltung sich als zu unserm Zwecke am meisten geeignet erweisen. Diese Organgruppen sind die im Dienste der Fortpflanzung umgewandelten Extremitäten oder Copulationsfüsse, deren Bedeutung für die Gruppirung VERHEFF in erster Linie erkannt und verfochten hat. Das Verständnis dieser Copulationsfüsse bietet allerdings manchmal Schwierigkeiten, weil sie meistens in weitgehender Weise durch Schwund oder Veränderung früher vorhandener Teile und durch Ausbildung von oft recht compliziert gestalteten Anhängen modifiziert wurden, so dass die Homologisierung und Deutung des Vorhandenen nur an der Hand grossen Vergleichsmateriales möglich ist.

Dieses Verständnis hat nun VERHEFF in seinem VIII. Aufsatze : Zur vergleichenden Morphologie, Phylogenie, Gruppen und Artsystematik der Chordeumiden. (Archiv für Naturgeschichte, 1899) in klarer und überzeugender Weise erschlossen, indem er an Beispielen klarlegt, wie durch schrittweise Umformung aus einem gewöhnlichen Lauffuss ein Copulationsfuss entstehen kann. Zugleich wird für die metamorphosierten Organe und ihre Bestandteile eine neue zweckmässige Benennung vorgeschlagen (z. B. Gonopoden für Copulationsfüsse) welche sich durch ihre Präcisität und Kürze zur allgemeinen Einführung empfiehlt.

Gattung Allochordeuma n. G.

In meinem ersten Beitrag beschrieb ich eine neue Chordeuma-Art, Chord. pallidum. Eine nochmalige Prüfung und Vergleichung mit den schon bekannten Chordeumince ergab, dass sie in keiner der bestehenden Gattungen untergebracht werden 
kann; dies veranlasst mich zur Aufstellung der neuen Gattung Allochordeuma mit folgender Diagnose ${ }^{1}$ :

Körper aus 30 Segmenten bestehend, hellgelb bis bräunlich, im äussern Habitus ähnlich Chord. silvestre.

Vier leicht trennbare Gonopodenpaare.

Hintere Gonopoden (2 Paar, 7 Segm.) mit einfacher, ziemlich breiter Ventralplatte. Hüften mit grossen, ausstülpbaren Hüftsäcken und mehreren verschiedenartig gestalteten Anhängen nebst Pseudoflagella. Femoroidelang, rinnenförmig, nicht gelenkig von der Hüfte abgesetzt.

Vordere Gonopoden (1 Paar, 7 Segm) : Ventralplatte hoch mit einem langen, mittleren Aufsatz und zwei seitlichen Stigmentaschen. Femoroide in Cheiroide umgewandelt, einfach, mit stumpflappigen Anhängen.

Vordere Nebengonopoden (2 Paar, 6 Segm.): Ventralplatte schmal, Hüften mit zwei langen, gebogenen Hörnern; Femora dick, kissenartig, beborstet; Tibialreste sehr klein.

Hintere Nebengonopoden (1 Paar, 8 Segm.): Ventralplatte schmal, Hüften gross, mit Hüftsäcken, Femora kurz, oben schräg abgestutzt. Tibiale gut ausgebildet, beborstet, Tarsalreste klein.

Allochordeuma pallidum Rothenb.

Fig. 7,8 und 10.

Meiner ersten Beschreibung füge ich hiemit eine bessere Darstellung des vordern Gonopodenpaares bei, Fig. 7. Eine Nachprüfung an frischem Material hat nämlich die interessante Thatsache ergeben, dass dieses Gonopodenpaar unzweifelhafte Cheiroide (Ch.) darstellt, d. h. Gebilde, welche aus der VerschmeIzung von Femoroiden und Tracheentaschen (Tr.) entstanden sind. Dieselben können durch die kräftige Muskulatur nach Art

1 Verheff stellt die Art zu Orthochordeuma (Archiv f. Naturgeschichte, 1900, Bd. I., Heft 3). Seine Publikation erschien, nachdem die erste Korrektur meiner Arbeit fertig war, daher kann ich jetzt nicht näher darauf eintreten. 
eines ungleicharmigen Hebels seitwärts bewegt werden. Cheiroide wurden bei den genauer bekannten Chordeumine, also den Gattungen Microchordeuma, Orthochordeuma, Chordeuma und Placodes nicht beobachtet und die beiden Gattungen Heterochordeuma Poc. und Pocokia Silv. sind in dieser Hinsicht nicht genügend beschrieben.

Der lange mediane Fortsatz der Ventralplatte endigt in einer langen, gedrehten Spitze ( $p r)$, welche die Enden der Cheiroide oft um ein geringes überragt. Seitlich bildet die Ventralplatte zwei Stigmengruben (St), deren oberer Rand sich zu einem niedrigen Höcker emporwölbt.

Für die Darstellung der übrigen Gonopoden verweise ich auf die Abbildungen in meiner frühern Arbeit (Revue suisse de Zoologie, Genf 1899).

Hintere Gonopoden (2 Paar, 7 Segm.): c. f. Fig. 17, l. c. In den Hüften liegen ausstülpbare Hüftsäcke. Femora ${ }^{1}$ rinnenförmig, fest mit den Hüften verbunden; diese besitzen drei Paar ververschieden gestaltete Anhänge, welche in den Femoralrinnen geborgen werden können; darunter ist ein Pseudoflagellum vorhanden, welches in Fig. 8 neu dargestellt ist; es wird von einem Kanal durchzogen, welcher am vielfach gefransten Ende etwas unterhalb der Spitze ausmündet. Der Kanal enthält eine granulöse Masse, wahrscheinlich das Sekret einer Drüse, die ich indes nicht nachweisen konnte. Die Beschaffenheit der übrigen Hüftanhänge ergibt sich aus Fig. 17, l. c.

Vordere Nebengonopoden (2 Paar, 6 Segm.): c. f., Fig. 15, l. c. Ventralplatte schmal, Hüften in einen langen, gebogenen, am Ende gabelig geteilten Fortsatz ausgezogen. Femora keulig, dick, kissenartig, beborstet, mit kleinen schwarz pigmentirten Tibialresten.

Hintere Nebengonopoden (1 Paar, 8 Segm.) : c. f., Fig. 18, l. c. und Fig. 10 in dieser Arbeit. Ventralplatte schmal, Hüften aufgedunsen, mit grossen Coxalsäcken Cos. Ein wohlabgesetzer

${ }^{1}$ Nach Verherf sind die Femora Teile der Hüften, Gonocoxide.

Rev. Suisse de Zool. T. 8. 1900. 
kurzer Femur (Fe Fig. 10) ist vorhanden, den ich zuerst übersehen und als blattartigen Hüftanhang bezeichnet hatte, da sein Vorderrand als runder Lappen vorragt. Tibiale (Ti) gut entwickelt, Tarsalreste rudimentär.

Fundorte: Bremgartenwald bei Bern, Schuls im Engadin.

Allochordeuma pallidum subsp. fulvum Rothenb.

Diese zuerst als Varietät beschriebene Form war auf ein einziges Exemplar gegründet. Seither fand ich die Tiere wieder mit denselben konstanten Abweichungen im Bau der Gonopoden, welche die Varietät als gute Unterart charakterisieren.

Für die bildlichen Darstellungen verweise ich auf meine Abbildungen im ersten Beitrag (Rev. suisse de Zool., 1899).

Vordere Gonopoden : c. f. Fig. 20, l. c. Dem medianen Processus der Ventralplatte fehlt die lange dünne Spitze; er ist abgerundet und erreicht nur die halbe Länge der Cheiroide. Die seitlichen Hörner der Ventralplatte sind dagegen länger und spitzer als bei pallidum. Die Cheiroide zeigen in der Mitte einen Vorsprung nach innen und die der basalen Hälfte aufsitzenden Lappen sind nicht stumpf gerundet, sondern spitz zahnartig.

Hintere Gonopoden : c. f. Fig. 17, l. c. Die Unterschiede gegenüber der Hauptform beschränken sich auf den mit 4 bezeichneten Hüftanhang, welcher bei pallidum lang und spitz, bei fulvum kurz und stumpf ist.

Vordere Gonopoden : c. f. Fig. 19, l. c. Hüften nicht direkt in die Anhänge verlängert; diese durch eine Naht abgesetzt, schlank, symmetrisch gebogen, mit einfacher, nicht geteilter Spitze. Femora im basalen Drittel stark verschmälert und desshalb schlanker als bei pallidum.

Die hinteren Nebengonopoden haben einen doppelt so langen Femur als bei der Hauptform.

Fundort: Bremgartenwald bei Bern. 


\section{Chordeuma nodulosum Verhœeff.}

Das Männchen dieser charakteristischen alpinen Form ist noch nicht gefunden.

Neuer Fundort: Engadin, ValTriazza, 2000 m., ein Weibchen mit 27 Segmenten.

\section{Gattung Orobainosoma Verh.}

Orobainosoma flavescens Latz., subsp. setosum n. subsp.

Fig. 5, 11, 14, 19.

Männchen $9^{\mathrm{mm}}$ lang, stark borstig, igelartig.

Vordere Gonopoden (Fig. 5) am Ende napfförmig erweitert und dadurch von der Hauptform auffällig unterschieden. Der Rand des Näpfchens ist mit Fransenbüscheln dicht besetzt; neben demselben mündet in einer Grube ein Drüsengang, welcher sich bis zu einer am Grunde liegenden Drüse mit Deutlichkeit verfolgen lässt. Die übrigen Teile des ganzen Organes stimmen mit denjenigen von flavescens überein. Die Ventralplatte erhebt sich in der Mitte zu einem am Ende abgerundeten und dort rinnig ausgehöhlten Aufsatz, dessen Gestaltung Fig. 11 als Lupenbild darstellt.

Hintere Gonopoden Fig. 14. Man vergleiche auch Fig. 13 und $13 a$ in meinem ersten Beitrag. Ventralplatte eine schmale gebogene Spange. Hüften breit, in der Mitte genähert mit rasch sich verschmälerndem Aufsatz, der in die lappigen Anhänge $L^{1}$ und $l^{1}$ ausläuft. Von den beiden ähnlichen Lappen $L$ und $l$ nahe der Basis der Hüfte verschmälert sich der grössere in einen langen, bandartigen Basalteil (linke Hälfte der Figur.) Nach aussen setzt sich an die Hüfte ein dickwalziger, beborsteter Femur mit Tibiale und kleinem Tarsalrest an.

Hintere Nebengonopoden (1 P. 8 Segm.) Fig. 19, mit grossen Hüftsäcken (vco.) und schräg nach innen gerichteten zugerundeten Hüftfortsätzen (pro.) welche eine lange Borste tragen. Die 
Beine normal aber schwach entwickelt. Am zweiten Beinpaar des 8. Segmentes sind ebenfalls kleine Hüftsäcke und Andeutungen von Hüftfortsätzen vorhanden.

Vorkommen: Bremgartenwald bei Bern.

Gattung Craspedosoma Verh.

Craspedosoma Rawlinsii Leach. subsp. simile Verh.

Fig. 12.

Bekanntlich sind bei Crasp. Rawlinsii die hintern Gonopoden verkümmert, dafür ist aber die Ventralplatte sehr stark entwickelt. Sie besteht aus einem Stücke, dessen Vorder- und Hinterrand sich zu je einem Querwulste erhebt; jeder dieser Wülste trägt drei in einer Querreihe stehende zapfenartige Erhöhungen, von welchen die mittlere die längste ist. Das ganze Gebilde variirt in der Form seiner einzelnen Bestandteile ausserordentlich, und zwar sind diese Veränderungen sowohl individueller als auch lokaler Art. Von Nyon am Genfersee besitze ich drei Männchen, deren Ventralplatte am Hinterrand fünf deutliche Höcker besitzt (Fig. 12), nämlich ausser den gewöhnlicher drei noch zwei kleinere zu beiden Seiten des Mittelhöckers. Die Tiere selbst zeichnen sich durch die lebhafte Färbung ihrer im übrigen typischen Zeichnung aus.

Craspedosoma Rawlinsii Leach, subsp. serratum n. subsp. Fig. 18.

Die unterscheidenden Merkmale dieser neuen Unterart liegen in der Gestaltung der Cheiroide, den Greifarmen der vordern Gonopoden. Die Abweichung in der Beschaffenheit dieser Organe ist eine so bedeutende und konstante, dass sie nicht in den Rahmen.einer Varietät gestellt werden kann. Dazu kommt, dass die betreffenden Tiere nach meinen bisherigen Erfahrungen durchaus Bewohner der südlichen Alpentäler, Wallis und Tessin, sind und also eine lokale Unterart repräsentiren. Cras- 
pedosoma Rawlinsii serratum unterscheidet sich von simile morphologisch nur durch die Cheiroide. Figur 18 stellt dieses Gebilde in seitlicher Ansicht, von aussen gesehen, dar. Es besteht der Hauptsache nach aus den drei Chitinlamellen $a, b$ und $c$. Die erste derselben (a) ist etwas kürzer als die beiden andern; ihr distaler Rand ist schwach gewölbt und vielfach ausgeschnitten; die Spitzen erinnern an die Zähne einer Säge. Der vordere Rand erhebt sich zu dem rinnenförmigen, nach vorn vorgewölbten Fortsatze $d$, senkt sich sodann zu dem entgegengesetzt gerichteten, ebenfalls rinnenförmigen Fortsatze $f$ und bildet in seinem weitern Verlaufe den Saum der beiden andern Chitinlamellen $b$ und $c$, von denen $b$ vielfach unregelmässig gezackt erscheint. Die beiden Blätter $b$ und $c$ schliessen eine tiefe, nach hinten offene Rinne ein, und ein ähnlicher Raum liegt zwischen den Lamellen $a$ und $b$. Bemerkt sei noch, dass der verschmälerte Teil des Cheiroides $(g)$ durch das Deckglas abgebogen und in eine etwas unnatürliche Lage gebracht wurde.

Vorkommen: Monte San Salvatore bei Lugano am Fusswege. Sitten und Siders im Wallis, je ein Exemplar.

\section{Gattung Atractosoma (Fanzago) Verhœeff.} Atractosoma meridionale Fanzago.

Fig. 1, 16.

Für die nähere Beschreibung dieser Art verweise ich auf LatzeLs classisches Werk. Auch die vordern Gonopoden sind von LATZEL richtig dargestellt worden. Indess gebeich in Fig. 16 eine ergänzende Abbildung derselben mit spezieller Berücksichtigung der Ventralplatte und Tracheentaschen.

Der auffallendste Bestandteil der vordern Gonopoden sind die zu einem mächtigen Syncoxid verschmolzenen Hüften Sync., deren Enden in je zwei gewundene Hörner ausgezogen sind, während ein drittes Paar gebogener Hörner $h$ seinen Ursprung nahe der Basis des Syncoxids nimmt. Die in zwei 
ungleich grosse Aeste ( $F$. und $f$.) gespaltenen einfachen Femoroide bilden mit den Tracheentaschen Tr einen am Syncoxid eingelenkten Hebel. Die Ventralplatte zeigt in der Mitte einen langen, schmalen, nach dem Körperinnern gerichteten Vorsprung $Z$, dem ein ähnlicher ( $k$ ) am distalen Ende des Syncoxids gegenüber steht.

Die hintern Gonopoden (2. Paar des 7. Segm.) Fig. 1, sind recht merkwürdig gestaltet durch die Beschaffenheit der Hüften und Femora. Die Hüften sind kurze hohle Cylinder, in welche die Femora vollständig zurückgebracht werden können, so dass sogar an deren Ansatzstelle eine kraterartige Einsenkung in der Hüfte entsteht. Da die Femora durchaus häutiger Natur sind, so werden sie jedenfalls auf gleiche Weise wie Hüftsäcke aus den Hüftkratern hervorgestülpt, nämlich durch Blutdruck. An den Femora sind wohlabgesetzte rudimentäre Tibien $T i$, mit einer Kralle zu bemerken. Die gesamte Extremität ist mit kurzen, kräftigen Borsten besetzt.

Die mit den Tracheentaschen verwachsene Ventralplatte trägt jederseits eine rundliche Grube.

Die Abbildung wurde nach zwei Präparaten hergestellt, von welchen das eine der rechten, das andere der linken Extremität als Vorlage diente.

Hüftsäcke oder Nebengonopoden habe ich bei $A$. meridionale nicht bemerkt.

Vorkommen: Unterengadin, Alp Lischanna, 1500 m. Ardez, $1400 \mathrm{~m}$., zusammen $3 \sigma^{\jmath}$.

Gattung Ceratosoma Verh.

Ceratosoma Caroli n. sp. ${ }^{1}$

Fig. 2, 4, 17, 22.

Länge $11 \mathrm{~mm}$. Körper aus 30 Segmenten bestehend, nach vorn stark verschmälert.

\footnotetext{
${ }^{1}$ Mit dieser Artbezeichnung möchte ich meinen Freund Dr J. CARL ehren, dem ich sämtliches Material aus dem Engadin verdanke.
} 
Rücken mit heller, vertiefter Längslinie. Seitliche Schilde der Segmente hoch angesetzt, stumpf, Oberseite blasig aufgetrieben mit den charakteristischen drei Borsten.

Farbe schmutzig erdbraun, Seiten und Bauch heller. Fühler lang und dünn, Augenfeld dreieckig schwarz, Ocellen zahlreich.

Männchen: Letztes Tarsale des 1. und 2. Beinpaares mit dem bekannten Borstenkamm; 3.-6. Paar an gleicher Stelle mit durchscheinenden, palissadenähnlichen Lamellen.

Nebengonopoden: Am Innenrand der Hüften des 7. Beinpaares stehen kleine, glasartig durchscheinende Wärzchen.

Die beiden Paare des 8. Ringes zeigen an den Hüften dieselben Wärzchen ; ausserdem sind sie mit Hüftsäcken versehen.

Zu eigentlichen Gonopoden sind die beiden Paare des 7. Segmentes umgestaltet.

Hintere Gonopoden: (2. P. 7. Segm.) Fig. 2. Tracheentaschen und Ventralplatte zu einem bogenförmigen Stücke verwachsen. Stigmen sind sichtbar. Die Ventralplatte läuft auf beiden Seiten in ein kurzes Hörnchen aus. Zwischen diesen Hörnchen sitzen die beiden fast ovalen, an der Spitze schwach beborsteten zweigliedrigen Beinrudimente mit schwarzen Pigmentflecken.

Vordere Gonopoden: (1. P. 7. Segm.) Fig. 4. Dieses Paar samt der dazugehörigen Ventralplatte ist ein so zartes, schwaches Gebilde, dass es schon durch den Druck des Deckglases breit gequetscht und zu jeder orientirenden Beobachtung unbrauchbar wird. Das Deckglas muss daher durch eine geeignete feste Unterlage (Glassplitter) gestützt werden, so dass das Präparat sozusagen frei im Glycerin schwimmt. Alsdann ergibt sich folgendes:

Die Ventralplatte, Fig. 22, stellt sich von der dem Körperinnern zugewendeten Seite gesehen als eine dünne Chitinlamelle dar, in welcher wir eine mittlere Längsleiste und von dieser ausgehend zwei Paar seitlicher Verdickungen bemerken. Vom hintern, caudalwärts gelegenen Ende der Längsleiste (E) ziehen 
zwei starke, divergirende Muskelbündel nach den vordern Ecken der Platte und von hier aus verlaufen zwei weitere Bündel der Vorderseite parallel nach der hier sehr starken medianen Längsrippe hin. Die Hinterseite der Platte ist bogig ausgeschnitten und die Ecken verlaufen in ziemlich lange Lappen, deren Ende umgeschlagen und zweizähnig ausgeschnitten ist. (Fig. 4,l).

Sieht man sich das Präparat von der andern Seite an, so bemerken wir auf der auf Fig. 22 mit $E$ bezeichneten Stelle, wo die Muskelbündel zusammentreten, zwei seitliche compliciert gestaltete Erhebungen, die man als die Hüften $C o$ ansprechen könnte, Fig. 4. An dem vielfach differenzirten Rande derselben unterscheiden wir folgende Teile: Auf dem runden Lappen $r$ zwei schlanke und eine stumpfe Spitze $s p$, eine tiefe Ausbuchtung $b$ und am Rande derselben wieder zwei feine Spitzen. Ein zweimal gebogener Wulst $t$ zieht sich vom Lappen $r$ nach der medianen Mittelrippe der Ventralplatte hinunter.

Etwas hinter den vordern Ecken der Ventralplatte nehmen die Cheiroide $G$ ihren Ursprung. Sie verlaufen zuerst quer nach der Mitte hin, biegen dann um und kommen mit den Endhälften parallel neben einander zu liegen. Das letzte Drittel ist gewunden und das Ende trägt einen langen und zwei kurze fingerförmige Fortsätze.

Die Tracheentaschen scheinen der Längsseite angeschmiegt ; ob dies ihre natürliche Lage ist, kann ich nicht entscheiden, weil ich kein Material mehr besitze.

Der Gonopodenring, Fig. 17, trägt am Rande seiner ventralen Oeffnung vorn einen kleinen geraden, hinten einen grossen gebogenen Fortsatz.

Vorkommen: Engadin, Schuls und Alp Tetan, 2 ơ und 2 q

Anmerkung: Ich stelle diese Art mit einigem Zweifel zur Gattung Ceratosoma. Sie zeigt nämlich mit zwei von ATTEms beschriebenen neuen Arten (AтTEms, Neues über palaarktische Myriapoden, Zool. Jahrbücher, 12. Band 1899) unzweifelhaft 
nahe Verwandtschaft. ATTEMs stellt seine Tiere aber zur Gattung Atractosoma. (A. condylocoxa und A. phyllophagum.) Es scheint mir jedoch, dass diese beiden Arten und meine neue Art besser bei Ceratosoma eingereiht würden, da die Aehnlichkeit der Copulationsfüsse zwischen Ceratosoma Caroli und Ceratosoma Apfelbecki ${ }^{1}$ viel grösser ist als beispielsweise diejenige von C. Caroli und Atractosoma meridionale.

\section{Gattung Trimerophoron n. g.}

Körper aus 30 Segmenten bestehend, mit seitlichen beuligen Auftreibungen, Craspedosomen-ähnlich.

Fühler lang, Augenfeld schwarz, dreieckig.

Am letzten Tarsale des 1. und 2. Beinpaares der Männchen ein Borstenkamm, an dessen Stellen bei den 5 folgenden Paaren ein Lamellenbesatz.

Hüftsäcke sind nur am 8. Beimpaar vorhanden. Hüften des 6. Beinpaares auffallend ausgezeichnet durch einen über das 7. Beinpaar hinübergreifenden Haken.

Vordere Gonopoden: Ventralplatte und Tracheentaschen sind drei isolirte, massive Stücke, die erstere T-förmig, ins Körperinnere zwischen die beiden Tracheentaschen verlagert, die letztern ohne Verbindung weder mit der Ventralplatte, noch mit den Femoroiden. Hüften ein Syncoxid. Die Femoroide sind längliche Chitinplatten mit Grannenbüschel.

Hintere Gonopoden mit dünnhäutiger Ventralplatte. Tracheentaschen mit den Hüften verwachsen. Femora undeutlich abgegliedert, mit Borsten und Papillenbesatz, schwache Tibialreste vorhanden.

Hüften des 1. Paares am 8. Segmente mit Hüftsäcken und Borsten und Papillenbesatz. Zugehörige Ventralplatte breit und hoch.

${ }^{1}$ Fig. 28 in : Verherf, Beiträge zur Kenntnis palæarkt. Myriap., Archiv f. Naturgesch., 1899, Band I, Heft 2. 
Trimerophoron grypischium n. sp.

Fig. $3,9,13,20,21$.

Länge 10-11 mm, 30 Segmente.

Farbe schmutzig hellbraun.

Ocellen deutlich, zahlreich, Augenfeld dreieckig, schwarz. Fühler lang, dünn; die drei letzten Glieder keulig verdickt. Körper Craspedosomen-ähnlich, ohne Kiele, aber mit beuligen Auftreibungen in den Oberflanken; je drei Borsten auf dem Rücken jedes Segmentes. Eine vertiefte Längslinie über die Rückenmitte ist vorhanden.

Die beiden ersten Beinpaare am letzten Tarsale mit dem bekannten Borstenkamm, die fünf folgenden mit einem Besatz durchscheinender Lamellen mit hakiger Spitze.

Die Gonopoden des 7. Segmentes sind verhältnismässig ziemlich einfach gestaltet.

Bei den Nebengonopoden finden wir, dass aussergewöhnlicher Weise nicht das hintere, sondern das vordere Paar des 6. Segmentes modificiert wurde.

Vordere Gonopoden (1. P. 7. Segm.) Fig. 21. Die ins Körperinnere verlagerte Ventralplatte $V$ bildet mit den beiden Tracheentaschen drei vollständig isolirte Stücke; sie hat die Form eines T, dessen Querbalken an den beiden Enden in gebogene, nach unten gerichtete Fortsätze und dessen Stützbalken spitz auslaufen. Die Tracheentaschen $\operatorname{Tr}$ stellen breite Chitinstücke dar, deren distales Ende in zwei Äste geteilt ist, wovon der eine schräg einwärts nach der Ventralplatte, der andere nach den Femoroiden hingeht und einen kleinen stumpfen Zahn trägt. Das Syncoxid Sync. lässt einen schmalen, querliegenden basalen und einen diesem aufsitzenden häutigen Teil erkennen, welcher als abgerundeter Hügel zwischen den Femoroiden liegt.

Die Femoroide $\mathrm{Fe}$ bilden zwei flache, breite Stücke mit zugerundetem Ende, welches sich seitwärts nach aussen in einen 
kurzen Schnabel verlängert; basalwärts von diesem Schnabel zeigt die hintere äussere Kante des Femoroids eine flache Einbuchtung, während die vordere Längskante als Besatz einen die Einbuchtung teilweise verdeckenden granulösen Saum trägt. Ein eigentümliches, federbuschartiges Gebilde ungefähr in der Mitte des Femoroids erinnert an den Grannenbüschel von Microbrachysoma alpestre.

Hintere Gonopoden (2. P. 7. Segm.) Fig. 9. Die Ventralplatte $V$ besteht aus einer weichen, dünnen Membran mit zwei stärkern Querstreifen. Die in der Mitte genäherten Hüften biegen am Grunde nach aussen um und bilden mit den Tracheentaschen Tr ein einziges Stück. Die verlängerten Hüften gehen ohne scharfe Absetzung in die mit Borsten und spitzen Papillen dicht besetzten Femora über, welchen lateral je ein kleines Tibiale ansitzt; sekundäre Wachstumsvorgänge mögen das Tibiale in seine seitliche Lage gedrängt haben.

$\mathrm{Zu}$ vordern Nebengonopoden ist nicht das 2., sondern das 1. Paar des 6 . Ringes differenzirt und durch die zweischenkligen Hüften ausgezeichnet. Es verdienen hervorgehoben zu werden:

a) Die Ventralplatte, Fig. 20. Ziemlich breit, eine mittlere und zwei seitliche Erhebungen; etwas innerhalb den letztern liegen die Eingänge St in die Tracheentaschen; diese sind mit der Ventralplatte verwachsen.

b) die Hüften, Fig. 13. Mit der Ventralplatte stehen sie nur durch Muskeln in Verbindung und lassen sich leicht von ihr trennen. Sie haben heberförmige Gestalt und bestehen aus den beiden Schenkeln $H$ und $G$ und dem mittlern Teile Co. Der dickere Schenkel $G$ liegt innerhalb der Ventralplatte im Körper neben der Tracheentasche und dient als Muskelansatzstelle. Der Rand der Ventralplatte kommt auf den nach vorn gerichteten Vorsprung $b$ zu liegen.

Der dünnere Schenkel $H$ greift wie ein Haken nach hinten hinüber über die Hüften des folgenden Beinpaares, so dass sein 
Ende zwischen dieses und die vordern Gonopoden zu stehen kommt. Die Umbiegungstelle zeigt Papillen und Borstenbesatz.

Der mittlere verbreiterte Teil Co trägt ein Laufbein, über dessen Beschaffenheit ich nichts angeben kann, weil nur der auf der Zeichnung sichtbare Femur vorhanden war; an demselben fallen die zahlreichen Papillen auf.

Die hintern Nebengonopoden (1. P. 8. Segm.) Fig. 3 sitzen auf einer breiten Ventralplatte, an welcher die Randverdickung, eine länglichrunde Verdickung in der Mitte und ein stumpfer, zwischen den Hüften liegender Fortsatz zu bemerken sind. Die Hüften zeigen grosse Hüftsäcke cos nebst Papillen- und Borstenbesatz. Auch hier waren die zugehörigen Laufbeine nicht mehr vorhanden, es kann daher über deren Beschaffenheit nichts gesagt werden.

Vorkommen: Ein einziges Männchen erhielt ich aus dem Val Triazza, Engadin durch Herrn Dr CARL.

Familie JULID A Brandt. Gattung Blaniulus Gerv. und Verh.

Eine Berichtigung über Blaniulus fimbriatus Rothenb. (wahrscheinlich = palmatus Némec) muss ich wegen Mangel an Vergleichsmaterial aus Böhmen für später aufschieben.

Gattung Julus Verh.

Julus belgicus Latzel var. gracilis Rothenb.

Syn. Julus albolineatus (Luc.) Verh.

Julus albovittatus Verh.

Julus gracilis Rothenb.

Die im Jahre 1874 in den «Comptes rendus de la société entomologique de Belgique» erschienene Mitteilung LatzeL's über Julus belgicus scheint längere Zeit in der deutschen Litteratur ziemlich unbekannt geblieben zu sein, wie die Synonyma beweisen, 
und war auch mir entgangen. Julus gracilis repräsentirt eine Varietät von Julus belgicus und unterscheidet sich von diesem durch die hinteren Klammerblätter, welche sich bei der Varietät gegen das Ende hin stark verbreitern und dort ein nach vorn gerichtetes vielzähniges Velum tragen. (Fig. 36 in meinem ersten Beitrag. Vergleiche damit Fig. 5, pag. 137 im Archiv für Naturgesch., I. Band 1898.)

Vorkommen : Umgebung von Bern. Sitten im Wallis, zahlreich am Mont Tourbillon, Nyon am Genfersee.

Julus nigrofuscus Verh.

Fundort: Engadin, Alp Fetan bei Schuls, 1500 m.

Die Abbildungen der Klammerblätter, welche VERHEFF in den «Verhandl. d. zool. bot. Gesellschaft in Wien 1894, » gegeben hat, sind in manchen Einzelheiten nicht ganz zutreffend, genügen indes zur Erkennung der Art vollständig.

Julus alpivagus Verh.

Fundorte: Engadin. Alp Fetan 2200 m., Tiere in Copula am 28. August. Schuls, am Waldrand. Versam im Bündner Rheintal.

$$
\text { Julus trilineatus (C. Koch.) Latzel. }
$$

Syn. J. silvivagus Verh.

Zahlreich unter moderndem Rebenlaub am Seeufer bei Lugano; Färbung typisch, Spitze des Velums manchmal geteilt.

\section{Julus Odieri Brölemann.}

Aus den Schweizeralpen nicht bekannt, wohl aber aus den cottischen Alpen und der Tarantaise.

Col Clapier, Mont Albergian, Col de la Rocheur, Col de la Vanoise, Lac de Tignes, überall häufig.

Julus alemannicus Verhœff.

Neuer Fundort: Cottische Alpen.

Col du Mont, var. simplex, mit vielfach zerschlitztem obern Velumrand. 
Col Saint Bertéliney.

Julus allobrogicus Brölemann.

Fundort: Cottische Alpen, Col Clapier.

\section{Schizophyllum sabulosum Latzel.}

Cottische Alpen: Col Clapier, Mont Cenis, Col Saint Bertéliney, Mont Albergian, Col de la Rocheur, Col de la Vanoise, Col du Mont.

\section{Schizophyllum mediterraneum Latzel.}

Diese Tiere sind in ihrem Vorkommen dadurch bemerkenswert, dass sie nicht an spezielle Schlupfwinkel, wie z. B. herumliegende Gesteinstrümmer sie bieten, gebunden sind. Der mit Gras bewachsene Boden von Wiesen und Weiden wird von ihnen sogar bevorzugt. Zwei Thatsachen sprechen für diese Meinung. Bei Orvin im Berner Jura fand ich Schizophyllum mediterraneum zahlreich im Sande der Landstrasse umherlaufend, und zwar bei heisser Mittagssonne. Ein genaueres Nachsehen zeigte, dass die Tierchen auch links und rechts der Strasse im Grase zahlreich vorhanden waren.

Merkwürdiger ist ein zweites Vorkommnis. Anfangs Oktober 1898 bestieg ich mit einem Freunde den Monte Bré bei Lugano. Vom Dörfchen Aldesago aus gingen wir in gerader Linie über den Westabhang dem Gipfel zu. Zwischen kleinen Aeckern zog sich ein Stück altes Wiesland in die Höhe, dessen kurzer Rasen von der Sonne verbrannt war. Hier fand sich nun unser Tier so massenhaft, dass es unmöglich war, den Fuss abzusetzen, ohne einige davon zu zertreten. Sie lagen im dürren Grase am Boden oder hatten sich um die Spitzen der Grashalme herum aufgeringelt. Etwa $100^{\mathrm{m}}$ höher hatte die Anzahl der Tiere bedeutend abgenommen, war jedoch immer noch gross. Die Untersuchung ergab, dass die Tiere $\mathrm{zu}^{2}{ }_{3}$ noch nicht geschlechtsreif waren. 


\section{Julus nitidus Verhøeff.}

Julus nitidus spinifera wurde als neue Unterart in meinem ersten Beitrag auf Grund von 6 genauer bezeichneten unterscheidenden Merkmalen (pag. 252) aufgestellt. Es hat sich aber erwiesen, dass diese vermeintlichen Unterschiede allen nitidus zukommen mit Ausnahme des zweiten Punktes. Mein Irrtum wurde durch die nicht vollständige erste Diagnose veranlasst; dieselbe ist daher durch Aufnahme der folgenden fünf Merkmale zu ergänzen.

1. Scheitelfurche vorhanden. (Punkt 2: Vordere Segmentteile nadelrissig, wird fallen gelassen, weil Angabe auf zu starker Vergrösserung beruhend.)

2. Erstes Beinpaar der Männchen an der Umbiegung oft eckig, meistens aber mit kegelförmigem Fortsatz.

3. Am 2. Beinpaar der Männchen sind zwei nach vorn gerichtete häutige Hüftfortsätze vorhanden.

4. Erste Spitze der Hinterblätter schwach gebogen, das Ende in mehrere (2-5) feine Spitzen geteilt.

5. Eine feine, durchsichtige Membran, deren oberer Rand mehrere feine Spitzchen bildet, verbindet die erste und zweite Spitze der hintern Klauenblätter.

Die Unterart spinifera ist also wieder einzuziehen.

\section{Brachyiulus littoralis Verhœff.}

Die erste aus der Schweiz bekannte Brachyiulus-Art. Wurde bisher häufig mit Brachyiulus pusillus Leach verwechselt, mit welchem littoralis in der Farbe und Zeichnung — eine feine schwarze Rückenlinie, daneben zwei breite gelbe Längsbänder, Flanken hell-bis dunkelbraun —übereinstimmt. Die Copulationsfüsse entsprechen durchaus der Abbildung, welche VERHeEFF im « Archiv für Naturgeschichte 1898 » geliefert hat. 
Vorkommen: Sonniger Abhang am Ufer des Genfersees bei Nyon unter Steinen, $10^{\jmath}$ und 4 Q.

\section{ZUSAMMENFASSUNG.}

Unsere Kenntnis der schweizerischen Myriapodenfauna erhält durch die voranstehenden Mitteilungen folgenden Zuwachs :

a) Bekannte, aber für das Gebiet neu nachgewiesene Arten sind die 7 folgenden :

Polydesmus coriaceus.

$$
\text { illyricus. }
$$$$
\text { subulifer. }
$$

Atractosoma meridionale.

Julus nigrofuscus.

$\gg$ trilineatus.

Brachyiulus littoralis.

b) Ueberhaupt neu beschrieben sind folgende 5 Arten und Unterarten :

Polydesmus subinteger Steckii.

Orobainosoma flavescens setosum.

Craspedosoma Rawlinsii serratum.

Ceratosoma Caroli.

Trimerophoron grypischium, zugleich Vertreter einer neuen Gattung.

c) Chordeuma pallidum wurde als Vertreter der neuen Gattung Allochordeuma erkannt ${ }^{1}$.

${ }^{1}$ Siehe Anmerkung pag. 176. 
TAFEL 13.

\section{FIGURENERKLÄRUNG}

Polydesmus subinteger Latz. Steckii n. subsp.

Fig. 15. Ein Gonopod in seitlicher Ansicht.

Polydesmus coriaceus Porat.

Fig. 6. Ein Gonopod eines Tieres von Sitten im Wallis.

Allochordeuma pallidum Rothenb.

Fig. 7. Vordere Gonopoden.

» 8. Pseudoflagellum als Hüftanhang des zweiten Gonopodenpaares.

" 10. Hälfte der hinteren Nebengonopoden.

Orobainosoma flavescens Latz. setosum n. subsp.

Fig. ̋. Hälfte der vordern Gonopoden.

» 11. Mittlerer Aufsatz der vorderen Ventralplatte, Lupenbild.

) 14. Hintere Gonopoden.

" 19. Hälfte der hinteren Nebengonopoden, Hüfte und Femur.

Craspedosoma Rawlinsii Leach.

Fig. 12. Hinterer Querwulst der hinteren Ventralplatte von C. Rawlinsii Leach simile Verh.

» 18. Cheiroid (Greifarm) von C. Rawlinsii Leach serratum n. subsp. Atractosoma meridionale Franzago.

Fig. 1. Hintere Gonopoden.

" 16. Hälfte der vorderen Gonopoden.

Ceratosoma Caroli n. sp.

Fig. 2. Hintere Gonopoden.

\4. Vordere Gonopoden.

» 17. Ventraler Rand des Gonopodenringes.

")2. Vordere Ventralplatte von innen.

Trimerophoron grypischium $\mathrm{n}$. sp.

Fig. 3. Hintere Nebengonopoden, Ventralplatte und Hüften.

»9. Hintere Gonopoden.

"13. Vordere Nebengonopoden ohne Ventralplatte.

"20. Ventralplatte der vorderen Nebengonopoden.

"21. Vordere Gonopoden. 


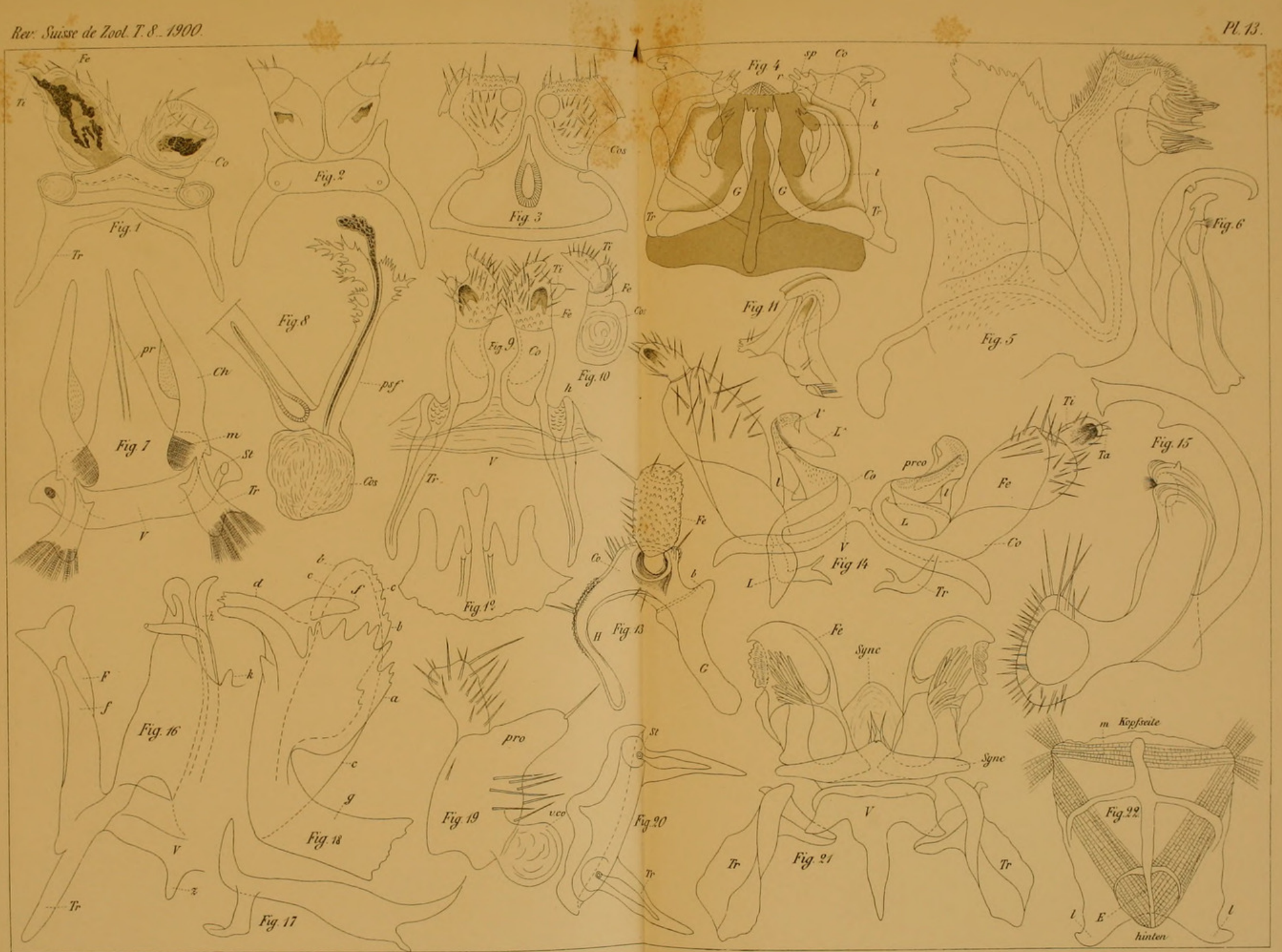

H Rothenisihior del

H. Rothenbüler - Diplopoda. 


\section{$2 \mathrm{BHL}$ Biodiversity Heritage Library}

Rothenbühler, Hans. 1900. "Zweiter Beitrag zur Kenntnis der

Diplopodenfauna der Schweiz." Revue suisse de zoologie 8(2), 167-192. https://doi.org/10.5962/bhl.part.10583.

View This Item Online: https://www.biodiversitylibrary.org/item/38141

DOI: https://doi.org/10.5962/bhl.part.10583

Permalink: https://www.biodiversitylibrary.org/partpdf/10583

\section{Holding Institution}

MBLWHOI Library

\section{Sponsored by}

MBLWHOI Library

\section{Copyright \& Reuse}

Copyright Status: NOT_IN_COPYRIGHT

This document was created from content at the Biodiversity Heritage Library, the world's largest open access digital library for biodiversity literature and archives. Visit BHL at https://www.biodiversitylibrary.org. 